\title{
Multiparameter Flow Cytometry Identification of Neoplastic Subclones: A New Biomarker in Monoclonal Gammopathy of Undetermined Significance and Multiple Myeloma
}

\author{
Fabián Tarín ${ }^{a}$ Francisco López-Castaño ${ }^{a}$ Carmen García-Hernández ${ }^{a}$ \\ Paola Beneit ${ }^{\mathrm{b}}$ Héctor Sarmiento ${ }^{\mathrm{a}}$ Pablo Manresa ${ }^{\mathrm{a}}$ Olga Alda $^{\mathrm{a}}$ \\ Blanca Villarrubia ${ }^{a}$ Margarita Blanes ${ }^{c}$ Javier Bernabéu ${ }^{c}$ Carmen Amorós ${ }^{d}$ \\ Sara Sánchez-Sánchez ${ }^{\mathrm{e}}$ Carmen Fernández-Miñano ${ }^{f}$ Francisco De Paz ${ }^{a}$ \\ José Verdú-Belmar ${ }^{\mathrm{a}}$ Pascual Marco ${ }^{\mathrm{a}}$ Estella Matutes ${ }^{\mathrm{g}}$

\begin{abstract}
${ }^{a}$ Department of Hematology, Hospital General Universitario de Alicante, Alicante, Spain; ${ }^{\text {b Department of }}$ Hematology, Hospital Universitario San Juan de Alicante, San Juan de Alicante, Spain; ' Department of Hematology,

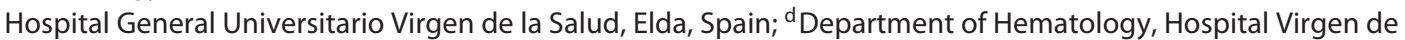
los Lirios, Alcoy, Spain; ${ }^{e}$ Department of Hematology, Hospital Marina Baixa, Villajoyosa, Spain; ${ }^{\mathrm{D}}$ Department of Hematology, Hospital Vega Baja, Orihuela, Spain; ${ }^{9}$ Department of Hematology, Hospital Clinic de Barcelona, Barcelona, Spain
\end{abstract}

\section{Keywords}

MGUS · Multiple myeloma · Flow cytometry $\cdot$ Neoplastic subclones

\begin{abstract}
Multiparameter flow cytometry (MFC)-based clonality assessment is a powerful method of diagnosis and follow-up in monoclonal gammopathy of undetermined significance (MGUS) and multiple myeloma (MM). However, the relevance of intraclonal heterogeneity in immunophenotypic studies remains poorly understood. The main objective of this work was to characterize the different immunophenotypic subclones in MGUS and MM patients and to investigate their correlation with disease stages. An 8-color MFC proto-
\end{abstract}

col with 17 markers was used to identify the subclones within the neoplastic compartment of 56 MGUS subjects, 151 newly diagnosed MM patients, 30 MM subjects in complete remission with detectable minimal residual disease, and 36 relapsed/refractory MM patients. Two or more clusters were observed in $>85 \%$ of MGUS subjects, $75 \%$ of stage I MM patients, and $<15 \%$ in stage III. Likewise, a significant correlation between the dominant subclone size, secondary cytogenetic features, and changes in the expression of CD27, CD44, and CD81 was detected. The loss of intraclonal equilibrium may be an important factor related with kinetics and risk of progression not well considered to date in MFC studies. The MFC strategy used in this work can provide useful biomarkers in MGUS and MM.

(c) 2018 S. Karger AG, Basel

\section{KARGER}

(C) 2018 S. Karger AG, Basel

E-Mail karger@karger.com

www.karger.com/aha
Fabián Tarín

Department of Hematology, Hospital General Universitario de Alicante

Avenida Pintor Baeza 11

ES-03010 Alicante (Spain)

E-Mail tarin_fab@gva.es 


\section{Introduction}

In recent years, multiparameter flow cytometry (MFC) has considerably increased its diagnostic relevance in monoclonal gammopathy of undetermined significance (MGUS) and multiple myeloma (MM). This technology allows the distinguishing of clonal neoplastic versus normal polyclonal plasma cells (PCs) with great sensitivity and specificity [1]. MFC-based clonality assessment is the most stringent method of diagnosis and follow-up, and provides very useful information to assess the risk of progression from MGUS to MM [2]. MFC also plays a fundamental role in the diagnosis of amyloidosis [3], staging of solitary bone plasmacytoma [4], evaluation of minimal residual disease (MRD) [5] and prognosis [6], and identification of new promising therapeutic targets in PC dyscrasias [7].

Intratumor heterogeneity is a widespread phenomenon of critical importance to understanding MGUS and MM pathogenesis [8-11]. The presence of subclonal clusters with different immunophenotypes and genotypes, and probably different sensitivity or resistance to treatment, has been clearly demonstrated [8-10]. However, the relevance of this finding in MFC studies remains poorly understood. The main objective of this study was to characterize the different immunophenotypic subclones in MGUS and MM patients, investigating their correlation with disease stages and whether the subclones remained stable or changed along the disease course.

\section{Patients and Methods}

Between March 2013 and June 2017, we used an 8-color MFC protocol to analyze the intratumoral phenotypic heterogeneity of 56 MGUS subjects, 151 newly diagnosed MM patients, $30 \mathrm{MM} \mathrm{pa-}$ tients who reached complete remission with detectable MRD (CR/ $\mathrm{MRD}+$ ), and $36 \mathrm{relapsed} /$ refractory MM patients defined according to IMWG criteria [12]. Likewise, comparison of the number and characteristics of subclones was made between the time of diagnosis and the first response evaluation, both in relapsed/refractory $\mathrm{MM}$ patients and $\mathrm{CR} / \mathrm{MRD}+$, to see possible changes in their immunophenotype.

Our strategy consisted in characterizing the clonal population through the EuroFlow PCD standardized panel [13], to later identify the subclones using four specifically designed 8-color combinations. For this, we used 17 monoclonal antibodies divided into 5 backbone markers placed in the same position to provide identical multidimensional localization of the target cells (CD38, CD138, CD19, CD81 and CD45) and 12 characterization markers (CD56, CD117, CD27, CD28, CD44, CD200, $\beta_{2}$-microglobulin, CD33, CD150, CD86, CD272 and CD52). The files were first merged and analyzed using new software tools based on principal component analysis and displayed by the automatic population separator (APS) graphical representation of the Infinicyt ${ }^{\mathrm{TM}}$ software (Cytognos). APS integrates all the parameters and defines the best possible separation between clusters, as well as the significance of each parameter in this separation.

\section{Results}

By analyzing the neoplastic compartment, two or more clusters were observed in the great majority $(>85 \%)$ of MGUS patients (Fig. 1a, 2a). On the other hand, this condition was present in only $42 \%$ of MM patients $(p<0.001)$, with the most common finding being a dominant subclone that represented $>75 \%$ of the neoplastic compartment (Fig. 1b, 2a). In both MGUS and MM, the top-parameters that best separated the different subclones were, in order of importance, CD81, CD56, CD44, CD28, CD27, CD117, CD19, CD200, CD45, CD138, and $\beta_{2^{-}}$ microglobulin.

Differences were also seen according to the stage of the disease in newly diagnosed MM. Thus, advanced DurieSalmon stages had fewer subclones detected $(p<0.01)$. Of stage I MM patients, $75 \%$ presented at least 2 subclones, as opposed to $<15 \%$ in stage III. This occurs similarly with other staging systems, such as the ISS and the R-ISS (Fig. 2b). In relapsed and refractory MM patients the dominant subclone virtually approached $100 \%$ of neoplastic PCs (Fig. 1c, 2c).

The immunophenotypic characteristics of the dominant clone were not clearly different between MGUS and newly diagnosed MM. Likewise, differences were also not observed between the different MM subtypes or clinical stages. However, when we compared the diagnosis and relapse, 31 out of 36 patients showed shifts of intensity of one or more markers restricted to the dominant subclone. The most frequent changes were the loss of CD27 intensity (26 patients) and increase of CD44 (23 patients) and CD81 (21 patients). Interestingly, these changes were not observed in 26 out of $30 \mathrm{CR} / \mathrm{MRD}+\mathrm{MM}$ patients in whom an intraclonal equilibrium similar to MGUS was partially restored (Fig. 1d, 2c; $p<0.01$ ).

Finally, in MM patients with secondary cytogenetic features (1q21+, 17p-, 6q-, and 8p-) [14] the dominant subclone was apparently higher compared to MM with only primary features (Fig. $2 \mathrm{~d} ; p<0.01$ ) and a particularly significant correlation between the percentage of PCs carrying 1q21+ and the dominant subclone size was demonstrated (Fig. 2e; $p<0.01$ ). 
Fig. 1. MFC analysis and subclones in MGUS and MM. a MGUS patient. Identification of polyclonal (blue) and neoplastic (red) PC clusters using APS. The different expression of three markers (CD81, CD45, and CD56) allows the identification of three different neoplastic subclones. b Newly diagnosed MM patient. Identification of a dominant subclone occupying $>90 \%$ of the neoplastic compartment. The different expression of three markers (CD56, CD81, and CD27) allows a minor secondary subclone to be distinguished. c Progressive MM patient. A large homogeneous dominant subclone can virtually approach $100 \%$ of all PCs. d CR/MRD+ MM patient. Similar to MGUS, the polyclonal PC cluster is clearly defined. The neoplastic cluster includes three different subclones with different expressions of CD81, CD27, and CD56.

Identification of Neoplastic Subclones in MGUS and Multiple Myeloma
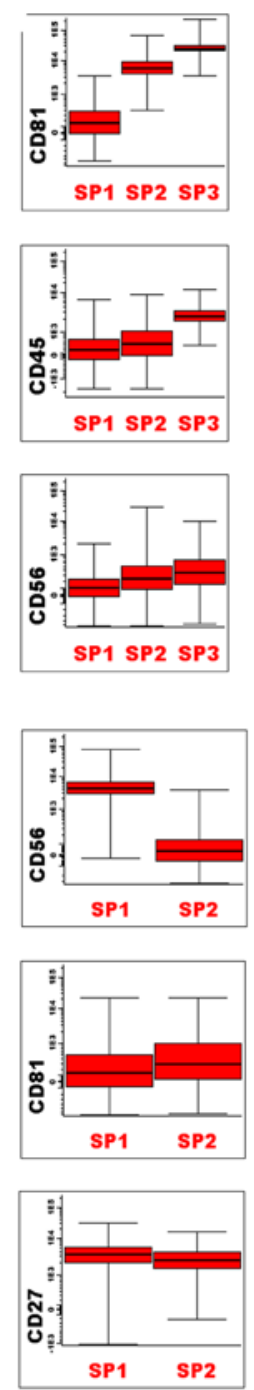

b
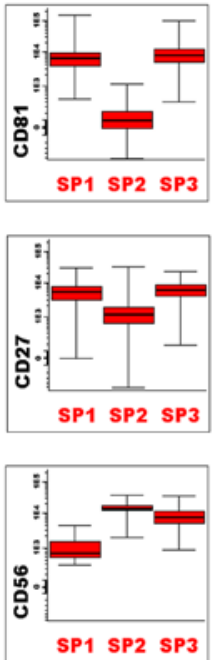
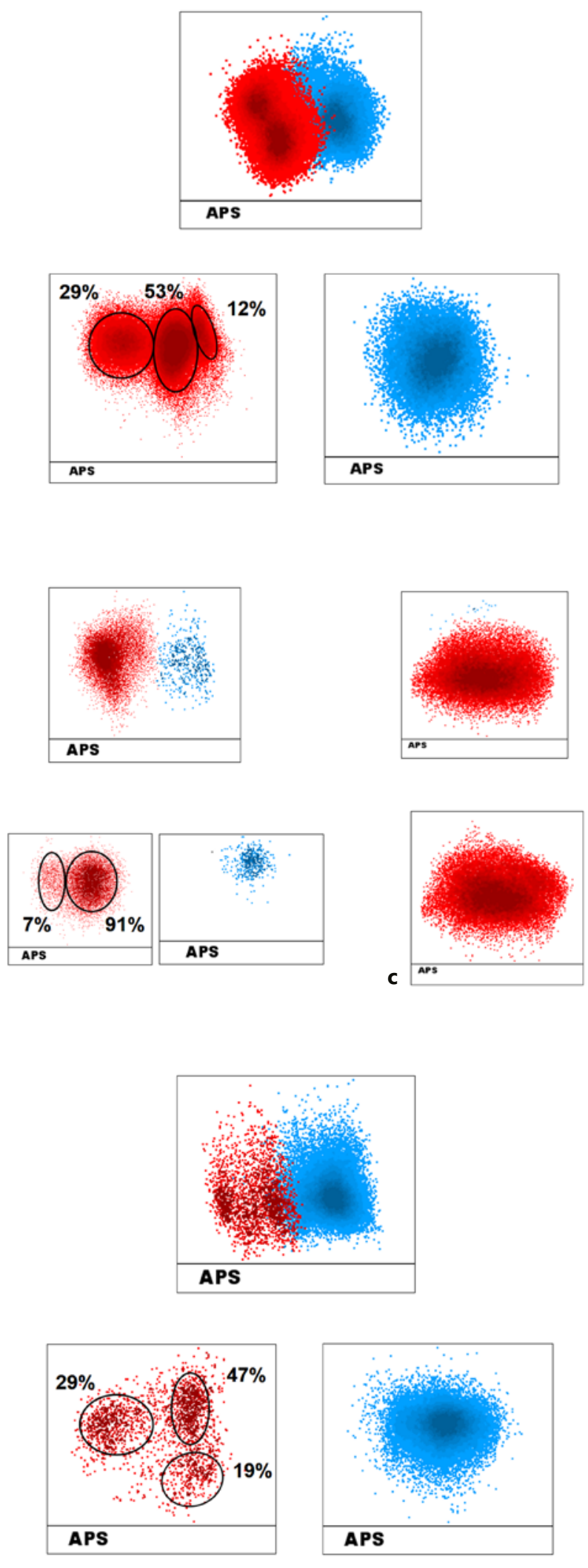


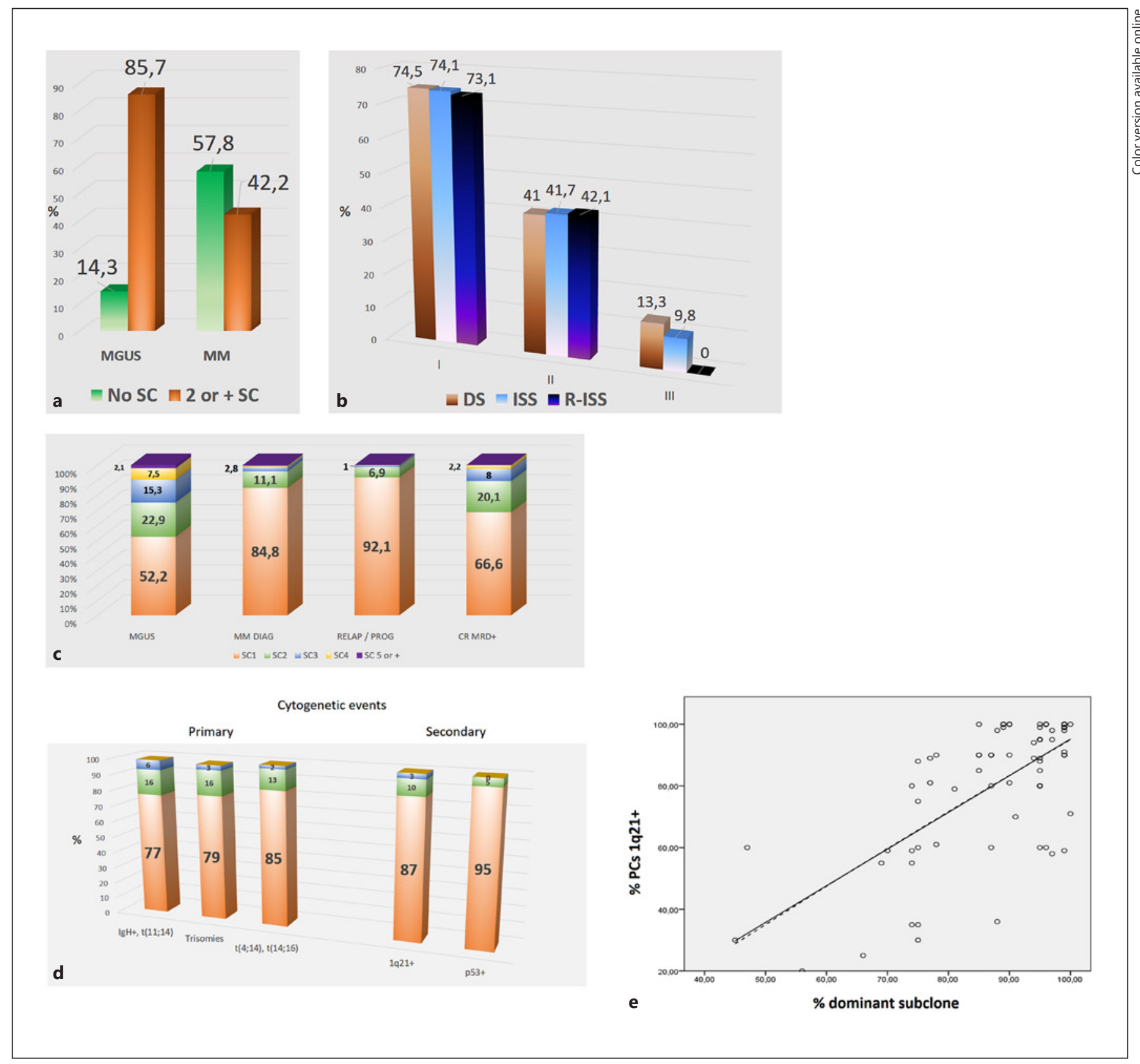

Fig. 2. Number and size of the different subclones related to the disease stage and cytogenetic events. a MGUS versus MM. The presence of subclones is more frequent in MGUS than in MM patients $\left(\chi^{2}=244.145, p<0.001\right)$. b Different MM stages. The progression to advanced Durie-Salmon, ISS, and R-ISS stages was closely related to the decrease in the number of detectable subclones $\left(\chi^{2}=44.122, p<0.01\right)$. c Intraclonal equilibrium in MGUS and MM. The number and size of subclones show relevant differences between MGUS and MM, probably reflecting the progressive loss of the intraclonal equilibrium. Interestingly, most CR/
MRD+ patients can recover a "MGUS-like" pattern (ANOVA $F=$ 26.473, $p<0.01$ ). d Intraclonal equilibrium and cytogenetics. In MM patients with secondary cytogenetic features, the dominant subclone size was higher compared to MM with only primary features, perhaps with the exception of the high-risk primary cytogenetic group (ANOVA $F=19.294, p<0.01$ ). e Intraclonal equilibrium and cytogenetics. High correlation between the percentage of PCs carrying 1q21+ and the dominant subclone size $(R=0.69, p<$ $0.01)$. 


\section{Discussion}

Several studies have highlighted the value of the ratio between aberrant and total PCs to establish the differential diagnosis and risk stratification in MGUS and MM [2]. Additionally, recent studies suggest that the loss of intraclonal equilibrium may be an important factor related to kinetics and risk of MGUS and MM [9]. Our study demonstrates that the neoplastic compartment is also very heterogeneous, revealing important and additional information, since the loss of intraclonal equilibrium may be an important factor related to kinetics and the risk of progression not well considered to date.

The presence of a large dominant subclone is a usual finding in MM patients indicating the progressive loss of tumor heterogeneity. This phenomenon seems to characterize especially advanced stages and relapsed/refractory patients. The correlation between the dominant subclone size and the percentage of PCs carrying 1q21+ reinforces this observation and strongly suggests that in many cases this disease progression is probably related to a competitive mechanism of dominancy of the $1 \mathrm{q} 21+$ subclone.

Likewise, other investigations have focused on the prognostic value of certain immunophenotypes, such as increased expression of CD19, CD20, CD44, and CD28, and lack of CD45, CD56, and CD117 [1, 6]. However, none of these markers have been proven by multivariate analysis to have independent prognostic significance. Interestingly, we have observed modifications in the expression of CD27, CD44, and CD81 throughout the evolution of the disease only in relapsed MM patients which are not present in residual cells from responsive $\mathrm{CR} / \mathrm{MRD}+\mathrm{pa}-$ tients. Changes in the expression of CD27, CD44, and CD81 are restricted to the dominant subclone and have been previously related with progressive disease $[1,6]$. Monitoring of all different detected subclones can reveal early data related to the risk of the subclonal dominancy and chemoresistance.

Some studies suggest that a better characterization of the neoplastic residual cells in CR/MRD+ MM patients may be relevant for clinical decisions [12]. Paiva et al. [15] provided very attractive data regarding the importance of the phenotypic and genetic characterization of these residual cells in order to understand chemoresistance. Our results show that a significant proportion of patients with $\mathrm{CR} / \mathrm{MRD}+$ can recover a subclonal pattern similar to MGUS. This new perspective can be useful to establish if different immunophenotypic features may identify highor low-risk subclones.

Identification of Neoplastic Subclones in MGUS and Multiple Myeloma
Finally, the discovery of new targets for tailored therapy play a major role in MM, and tools for their fast and reliable detection are essential $[1,8]$. Our results show that some makers (CD81, CD27, CD56, CD28, and CD44) have a variable expression and therefore a tendency to differentiate the subclones. Since they are probably implicated in the intraclonal heterogeneity, these findings must be considered when evaluating therapeutic strategies in order to avoid selecting initially minor subclones resistant to tailored therapy.

In conclusion, most of the existing diagnostic MFC algorithms based on "single-clone" models do not allow the ascertainment of the contributions of different subclones, implying a significant loss of relevant information. The MFC methodology used in our work allows the fast quantification and characterization of the different immunophenotypic subclones in MGUS and MM, and could modify the current paradigm of interpretation of the immunophenotypic features, providing new promising biomarkers in MGUS and MM.

\section{Statement of Ethics}

Subjects (or their parents or guardians) gave their written informed consent for inclusion in the study. The study protocol was approved by the research institute's committee on human research.

\section{Disclosure Statement}

The authors have no conflicts of interest to declare.

\section{Funding Sources}

This work was supported by research funding from Instituto de Investigación Sanitaria y Biomédica de Alicante (ISABIAL).

\section{Author Contributions}

Fabián Tarín, principal investigator: study concept and design; preparation, creation, and presentation of the work. Francisco López-Castaño and Paola Beneit: preparation, creation and presentation of the work; data collection and analysis. Héctor Sarmiento, Pablo Manresa, Margarita Blanes, Blanca Villarrubia, Javier Bernabéu, Carmen Amorós, Sara Sánchez-Sánchez, Carmen Fernández-Miñano, Francisco De Paz, and José Verdú-Belmar: data collection and analysis. Olga Alda, Pascual Marco, and Estella Matutes: critical review for intellectual content. 


\section{References}

1 Raja KR, Kovarova L, Hajek R. Review of phenotypic markers used in flow cytometric analysis of MGUS and MM, and applicability of flow cytometry in other plasma cell disorders. Br J Haematol. 2010 May; 149(3):334-51.

2 Pérez-Persona E, Mateo G, García-Sanz R, Mateos MV, de Las Heras N, de Coca AG, et al. Risk of progression in smouldering myeloma and monoclonal gammopathies of unknown significance: comparative analysis of the evolution of monoclonal component and multiparameter flow cytometry of bone marrow plasma cells. Br J Haematol. 2010 Jan; 148(1):110-4.

3 Paiva B, Vídriales MB, Pérez JJ, López-Berges MC, García-Sanz R, Ocio EM, et al. The clinical utility and prognostic value of multiparameter flow cytometry immunophenotyping in light-chain amyloidosis. Blood. 2011 Mar; 117(13):3613-6.

4 Paiva B, Chandia M, Vidriales MB, Colado E, Caballero-Velázquez $\mathrm{T}$, Escalante $\mathrm{F}$, et al. Multiparameter flow cytometry for staging of solitary bone plasmacytoma: new criteria for risk of progression to myeloma. Blood. 2014 Aug;124(8):1300-3.

5 Flores-Montero J, Sanoja-Flores L, Paiva B, Puig N, García-Sánchez O, Böttcher S, et al. Next Generation Flow for highly sensitive and standardized detection of minimal residual disease in multiple myeloma. Leukemia. 2017 Oct;31(10):2094-103.
6 Arana P, Paiva B, Cedena MT, Puig N, Cordon L, Vidriales MB, et al. Prognostic value of antigen expression in multiple myeloma: A PETHEMA/GEM study on 1,265 patients enrolled in four consecutive clinical trials. Leukemia. 2018 Apr;32(4):971-8.

7 Lisenko K, Schönland S, Hegenbart U, Wallenwein K, Braun U, Mai EK, et al. Potential therapeutic targets in plasma cell disorders: A flow cytometry study. Cytometry B Clin Cytom. 2017 Mar;92(2):145-52.

8 Brioli A, Melchor L, Cavo M, Morgan GJ. The impact of intra-clonal heterogeneity on the treatment of multiple myeloma. Br J Haematol. 2014 May;165(4):441-54.

9 Paíno T, Paiva B, Sayagués JM, Mota I, Carvalheiro T, Corchete LA, et al. Phenotypic identification of subclones in multiple myeloma with different chemoresistant, cytogenetic and clonogenic potential. Leukemia. 2015 May;29(5):1186-94.

10 Bolli N, Avet-Loiseau H, Wedge DC, Van Loo $\mathrm{P}$, Alexandrov LB, Martincorena I, et al. Heterogeneity of genomic evolution and mutational profiles in multiple myeloma. Nat Commun. 2014;5(1):2997.

11 Lohr JG, Stojanov P, Carter SL, Cruz-Gordillo P, Lawrence MS, Auclair D, et al.; Multiple Myeloma Research Consortium. Widespread genetic heterogeneity in multiple myeloma: implications for targeted therapy. Cancer Cell. 2014 Jan;25(1):91-101.
12 Anderson KC, Auclair D, Kelloff GJ, Sigman CC, Avet-Loiseau H, Farrell AT, et al. The Role of Minimal Residual Disease Testing in Myeloma Treatment Selection and Drug Development: Current Value and Future Applications. Clin Cancer Res. 2017 Aug;23(15): 3980-93.

13 van Dongen JJ, Lhermitte L, Böttcher S, Almeida J, van der Velden VH, Flores-Montero J, et al.; EuroFlow Consortium (EU-FP6, LSHB-CT-2006-018708). EuroFlow antibody panels for standardized n-dimensional flow cytometric immunophenotyping of normal, reactive and malignant leukocytes. Leukemia. 2012 Sep;26(9):1908-75.

14 Corre J, Munshi N, Avet-Loiseau H. Genetics of multiple myeloma: another heterogeneity level? Blood. 2015 Mar;125(12):1870-6.

15 Paiva B, Corchete LA, Vidriales MB, Puig N, Maiso P, Rodriguez I, et al.; Spanish Myeloma Group / Program for the Study of Malignant Blood Diseases Therapeutics (GEM / PETHEMA) Cooperative Study Groups. Phenotypic and genomic analysis of multiple myeloma minimal residual disease tumor cells: a new model to understand chemoresistance. Blood. 2016 Apr;127(15):1896-906. 